\title{
Tangence
}

\section{Les deux vies de Saint-Hyacinthe : dans les marges du Dr Mathanasius}

\section{Éric Méchoulan}

Numéro 57, mai 1998

Littérateurs atypiques et penseurs irréguliers

URI : https://id.erudit.org/iderudit/025966ar

DOI : https://doi.org/10.7202/025966ar

Aller au sommaire du numéro

Éditeur(s)

Tangence

ISSN

0226-9554 (imprimé)

1710-0305 (numérique)

Découvrir la revue

Citer cet article

Méchoulan, É. (1998). Les deux vies de Saint-Hyacinthe : dans les marges du Dr Mathanasius. Tangence, (57), 23-39. https://doi.org/10.7202/025966ar d'utilisation que vous pouvez consulter en ligne.

https://apropos.erudit.org/fr/usagers/politique-dutilisation/ 


\section{Les deux vies de Saint-Hyacinthe: dans les marges du Dr Mathanasius Éric Méchoulan, Université de Montréal}

De Saint-Hyacinthe, on ne se souvient plus guère que du Chef d'œuvre d'un inconnu qu'il publie anonymement en 1714. Dans la réédition qu'il en a récemment faite, Henri Duranton affirme que "ce que l'on connaît de Thémiseul de Saint-Hyacinthe [...] dresse le portrait d'un marginal qui, sans doute malgré lui, a vécu difficilement, hors des circuits traditionnels. " ${ }^{1}$ La vie de Saint-Hyacinthe semble en effet témoigner de son appartenance à cette "gueuserie" littéraire qui commence à envahir la République des Lettres au début du XviI ${ }^{\mathrm{e}}$ siècle. Ainsi M. des Sablons rapporte-t-il que Voltaire, dans ses Lettres secrettes, fait de SaintHyacinthe l'aimable portrait que voici : “infâme escroc \& sot plagiaire, voilà l'histoire de ses mœurs \& de son esprit. Il a été Moine, Soldat, Libraire, Marchand de Caffé, \& vit aujourd'hui du profit du Biribi. [...] C'est un de ceux qui déshonorent le plus les Lettres \& l'humanité. Il n'a guere vêcu à Londres que de mes aumônes \& de ses libelles. " 2 Saint-Hyacinthe apparaît alors comme un excellent exemple de "marginalité" ou d'“atypicité littéraire". Pourtant le même Voltaire publie, en 1768 , sous le nom de "Mr de St. Hiacinte" son Dîner du Comte de Boulainvilliers et il lui attribue, de façon apparemment élogieuse, Le militaire philosophe. C'est se servir, bien sûr, de la réputation de philosophe déiste, anti-religieux, de Saint-Hyacinthe, mais il s'agit aussi d'une marque de légitimation implicite de l'écrivain et de sa reconnaissance au sein de la République des lettres ${ }^{3}$. Où se situe donc Saint-Hyacinthe?

1 Thémiseul de Saint-Hyacinthe, Le cbef-d'cuvre d'un inconnu, présenté et annoté par Henri Duranton, Paris, Éd. du CNRS, 1991, p. 9.

2 M. des Sablons, Les grands bommes vengés, ou Examen des jugements portés par $M$. de Voltaire, \& par quelques autres pbilosopbes, sur plusieurs Hommes célebres..., Amsterdam, Jean-Marie Barret, 1769, vol. I, p. 190.

3 Dans une lettre à Saurin (5 février 1768, Best 13806), Voltaire écrit: "Le dîner dont vous me parlez est sûrement de Saint-Hyacinthe. On a de lui un Militaire philosophe qui est beaucoup plus fort et très bien écrit ", cité par Roland 
24

Thémiseul de Saint-Hyacinthe est né Hyacinte Cordonnier, d'une famille champenoise d'officiers appartenant aux Orléans (la mère de Hyacinte recevra une pension de Monsieur après la mort de son mari ${ }^{4}$ ). La rumeur voudrait qu'il soit en fait le fils de Bossuet, mais il s'agit d'une rumeur très tardive, dont on ne sait même si elle vint jamais jusqu'à lui. Il fait ses études à Troyes, chez les Oratoriens, quitte cependant l'école pendant son année d'humanités (sans que l'on sache exactement pour quelle raison ou quel scandale interne, mais on suppose généralement un conflit avec ses maîtres). Son esprit indépendant se manifesterait donc par ce premier coup d'éclat. Il étudie seul et participe à la vie mondaine de Troyes, ce qui lui vaut d'écrire, dans la tradition de l'esprit galant, quelques pièces qu'il publiera beaucoup plus tard sous le joli titre de "Pièces échappées du feu", mais qui ont eu pour prime nom "Polissoniana" (par où l'on saisit une dimension ludique, voire gaudriolesque, dont il ne se départira jamais tout à fait). Il revient chez les Oratoriens pour finir ses études, obtient un brevet d'officier subalterne et part, en 1703, prendre part à la Guerre de Succession d'Espagne. Mais il est vite capturé à Blenheim où le duc de Marlborough, sans attendre ni Pâques ni la Trinité, écrase les Français. Ce sont alors trois années de captivité en Hollande, ce qui signifie beaucoup de bières, de "tabagies", de lectures et de conversations (sans compter quelques "gentilles allemandes" comme il le dit).

De retour à Troyes, il renoue avec sa modeste vie mondaine qu'il doit interrompre en 1711 à cause d'un scandale dont, encore une fois, on ne sait pas grand chose, sinon qu'il devait toucher une jeune fille, peut-être noble, peut-être même une religieuse. Il repart donc pour la Hollande où deux crises le touchent profondément: une crise mystique (qui fait du catholique peu pratiquant qu'il était un déiste proche de l'athéisme, malgré un dialogue fervent avec le Père Quesnel) et une crise d'asthme (qui rendra sa santé pour toujours fragile). C'est à La Haye qu'il crée avec

Mortier dans son édition du Militaire philosophe qu'il public sous le titre, plus juste, de Difficultés sur la religion proposées au Père Malebrancbe, Bruxelles, Presses universitaires de Bruxelles, 1970. Roland Mortier conteste l'attribution de ce texte à Saint-Hyacinthe avec, semble-t-il, de bonnes raisons.

4 Je renvoie à l'excellente biographie faite par Élisabeth Carrayol, "Thémiseul de Saint-Hyacinthe, 1684-1746", dans Studies on Voltaire and the Eigbteenth Century, vol. 221, Oxford, 1984. 
quelques amis un Journal littéraire, puis réédite et préface le Traité du Poëme Epique, du Père Le Bossu, en réponse à une commande de libraire, enfin publie son Chef d'oeuvre d'un inconnu qui tient de la pochade et s'inscrit dans la longue lignée des pédants moqués de Janotus de Bragmardo et autres Hortensius jusqu'à Pangloss. Le fait de tomber en pleine résurgence de la Querelle des anciens et modernes lui assure un certain succès ${ }^{5}$. Il revient alors en France, publie des Mémoires littéraires où il se plaît à de multiples digressions à partir de sujets variés, mène une vie mondaine à Paris, monte un autre journal avec Lévesque de Burigny et Prosper Marchand (l'Europe savante), mais doit de nouveau retourner en Hollande à partir de 1720 pour des raisons de santé. Il y traduit la première partie de Robinson Crusoe et s'y fait, à nouveau, rattraper par le scandale: amoureux d'une jeune fille noble, riche et belle, la légende veut qu'il lui ait demandé de l'enlever, lui, afin d'éviter les poursuites judiciaires. Il l'épouse pour finir et, tous deux vont vivre en Angleterre où, grâce à la fortune de sa femme, Saint-Hyacinthe est, pour la première fois, financièrement à l'aise. Cependant diverses opérations malheureuses les replongent dans la gêne et, en 1732, il doit fuir à Paris des créanciers devenus trop pressants. Il y survit d'activités littéraires variées et d'appuis ponctuels dans les milieux mondains qu'il fréquente, mais il est mêlé au scandale de la Voltairomanie de l'abbé Desfontaines, dans la mesure où il avait inséré un épisode peu à la gloire de Voltaire (sans en mentionner le nom) dans sa Déification d'Aristarchus Masso (satire d'un journaliste qu'il exécrait), que Desfontaines récupère comme un témoignage de la veulerie de Voltaire. Plus grave, en 1740 , alors qu'il est en voyage

5 "C'étoit précisément dans le temps qu'il y avoit à Paris une dispute très-animée sur la comparaison des Anciens avec les Modernes. Les partisans de l'antiquité prêtoient au ridicule par leur exagération en faveur de ceux à qui ils donnoient la préférence, \& par le peu de justice qu'ils rendoient aux bons ecrivains de notre siecle. Cette partialité fut loccasion du Livre intitulé : Le Chef-d'cuvre d'un Inconnu, par Matbanasius [...]. Ce joli Ouvrage eut le plus grand succès: Paris en fut enthousiasmé pendant quelque tems; \& on le lisoit avec d'autant plus de plaisir, qu'outre que les commentateurs passionnés des Anciens y étoient toumés dans le plus grand ridicule, par l'imitation parfaite que l'Auteur avoit faite de leur méthode dans l'explication des Ecrivains de l'antiquité, on y trouvoit quelques traits assez plaisans qui avoient rapport aux Jésuites \& à la Bulle Unigenitus, qui causoit pour lors les plus grandes disputes, \& qui souffroit beaucoup de contradiction. "Lettre de $M$. de Burigny à $M$. l'Abbé Mercier sur les démêlés de $M$. de Voltaire avec $M$. de Saint-Hyacintbe..., Londres, Paris, Valade, 1780, p. 2-3. 
pour récupérer un héritage de son épouse, sa fille aînée se laisse enlever et mettre dans un couvent pour y être éduquée religieusement - ce scandale l'oblige à s'exiler encore une fois en Hollande où sa femme meurt deux ans plus tard et où il s'éteint lui-même en 1746 dans la plus totale misère.

Existence, donc, de bric et de broc qui va d'exil en scandale, de provisoires réussites en retombées chaque fois plus radicales, d'une polygraphie mondaine et enjouée à une philosophie morale et sérieuse, qui semble bien témoigner du statut incertain de ce que Darnton (et Goulemot à sa suite) appelle, de façon sciemment anachronique, la "bohème littéraire" et que l'on pourrait rendre d'un vocable plus adéquat à l'époque par "les aventuriers littéraires " 6 . De la même façon que se développent les possibilités, pour les cadets sans fortune et les petits nobles désargentés, de parcourir le monde et de chercher une réussite hors des limites de l'Europe, il est devenu courant, pour nombre de jeunes gens bien éduqués par un système d'enseignement en plein essor, de se lancer dans l'aventure des Lettres en tentant de convertir leur capital de savoir en capital à la fois symbolique et financier (pour parler comme Bourdieu), dans la mesure où la carrière des lettres offre désormais les deux (au moins pour quelques-uns). C'est en quoi Jean Marie Goulemot a sans doute raison d'affirmer que "l'opposition réelle ne serait donc pas entre partisans et adversaires des Lumières, mais entre écrivains établis - sorte d'establishment de la littérature - et bohème malheureuse " ${ }^{7}$. Mais cela permet-il vraiment de rendre compte de la "carrière" d'un Saint-Hyacinthe, dans la mesure où l'on pourrait raconter son histoire de manière presque exactement inverse?

Seconde vie, donc, de Saint-Hyacinthe. Bonne éducation chez les oratoriens (sciences, histoire, langues vivantes, latin, un peu de grec et une initiation philosophique surtout platono-augustinienne, mais teintée d'un brin de cartésianisme), en prise sur l'actualité. Il apparaît comme un "eleganti ingenio adulescens", autrement dit un jeune homme à l'esprit distingué. De fait, une fois dans le monde, il s'affirme comme bel esprit, à l'aise dans les jeux littéraires des salons provinciaux. Sa captivité en Hollande lui permet

\footnotetext{
6 La Barre de Beaumarchais publie à La Haye en 1733 un ouvrage intitulé Secret qu'ont les aventuriers littéraires de passer pour Scavans.

7 Jean Marie Goulemot et Daniel Oster, Gens de Lettres, écrivains et bohèmes: l'imaginaire littéraire, 1630-1900, Paris, Minerve, 1992, p. 86-87.
} 
d'enrichir considérablement une culture générale déjà conséquente dont on verra des traces dans le Journal littéraire qu'il fonde avec des personnalités aussi marquantes que S'Gravesande et Sallengre, et qui alimente jusque dans la parodie le Chef d'cuvre d'un inconnu. Ce texte témoigne en effet d'une aisance à manipuler la tradition savante et la rhétorique de l'école, en même temps qu'il s'inscrit dans le jeu mondain du portrait à charge et de la satire enjouée. Son succès s'explique non seulement par l'effet d'actualité (tomber en pleine querelle d'Homère), ce qui dénote au moins un sens aiguisé des courants contemporains, mais aussi par la qualité intrinsèque de la satire et le ton distingué du badinage érudit qui, à la fois, met à la portée des mondains des questions d'école et leur donne un sentiment de supériorité sur les savants. L'ouvrage, publié anonymement, est d'abord attribué à nul autre que Crousaz ou Fontenelle: c'est dire la qualité d'écriture et le savoir engagé dans cette "pochade" de jeunesse. Ce n'est donc pas hasard ou chance (du débutant) si le texte ne cesse d'être réédité (trois éditions pirates dès l'année de parution - ce qui est un signe du succès du texte qui ne trompe pas - puis, officiellement, en 1716, 1728, 1732, 1744, 1745, 1752, deux éditions en 1758, et l'édition Leschevin de 1807 qui fait autorité). Daniel Mornet dans son enquête sur les bibliothèques des particuliers recense 129 exemplaires du Chef d'ouvre (contre un exemplaire du Contrat social), ce qui le place au quatorzième rang, avant tous les romans! Le $\mathrm{D}^{\mathrm{r}}$ Mathanasius devient le personnage même de l'érudit prétentieux et vain qui fait de l'explication d'un texte tout simple de la tradition populaire un prétexte à délire savant $^{8}$. (En un sens, Mathanasius nous guette tous!)

Après ce succès, sa venue à Paris l'impose dans les principaux cercles mondains, soutenu de plus qu'il est par le Régent (puisque sa famille, traditionnellement, est dans la clientèle des

8 lisant la strophe quatre, par exemple, "Le galant qui fut honnête / Droit se coucha,? Entre les bras de sa belle / Se reposa. / Ah! je n'ai pas perdu mes peines, / Aussi mes pas, / Puisque je tiens celle que j'aime / Entre mes bras ", Mathanasius fait un sort au "Ah": "Que cet Ah! est beau! qu'il est éloquent! qu'il exprime bien que Colin était entièrement pénétré de son bonheur! Ah! est une voix de Iá nature, qui marque cette dilatation de coeur que causent les grandes passions. Cela est si vrai que toutes les nations du monde, les Hébreux, les Turcs, les Chinois, les Iroquois, les Français, les Anglais, les Hollandais même ont cette exclamation. Mais qu'on peut bien appliçuer ici à Colin ces deux vers de Pindare: Le vainqueur jouit d'une tranquillité plus douce que le miel. Etc.! 
Orléans). Il a d'ailleurs suffisamment de crédit pour avoir été chargé de justifier la politique française face aux menées espagnoles dans des Entretiens sur les entreprises de l'Espagne. Il ne s'en tient pourtant pas à la simple actualité et en profite pour réfléchir sur la société et l'État. Il traite des problèmes entre France, Espagne et Angleterre, par faits et analyses dans un premier entretien, par principes méthodiques dans le second, de manière à fonder en raison la compréhension des circonstances historiques et de l'évolution des États. Il glisse d'ailleurs, au passage, une critique des manières purement mondaines, ne pouvant se satisfaire (déjà en "philosophe des Lumières") de l'ignorance des principes sociaux et du mépris du bien commun:

comme ce n'est point la Mode en France de travailler à se rendre utile à l'Etat, mais seulement à soi même, nous ne confondons point le bien public avec le nôtre, deux choses cependant qui ne devroient jamais être desunies. On diroit en vain à nos jeunes Seigneurs: Apprenez à distinguer ce qui est vraiment juste d'avec ce qui n'a que l'Apparence de la justice. Approfondissez le Droit Public. Instruisez vous des Loix \& des Coutumes de vos Provinces. Apprenez ce que c'est que Finance, Ce que c'est que Commerce, Sçachez ou peuvent aller les forces de l'Etat soit par Mer, soit par Terre, Sçachez quelles sont celles des autres Etats, Voyagez, non pour donner aux Etrangers des exemples d'Etourderie \& de Presomption, mais pour apprendre leurs Mours \& leurs Loix, \& pour voir ce que les Arts produisent chez eux de commode \& d'utile. Quand nos jeunes gens ont lu les Poëtes, quelques Memoires Modernes, qu'ils savent sur le bout du doigt nos Tragedies, nos Operas, l'Histoire Scandaleuse de Paris, qu'ils ont une bonne provision d'Airs Nouveaux, qu'ils brillent dans un Cercle de femmes, qu'ils se font voir au spectacle parez comme des Coquettes, qu'ils savent tenir table pendant cinq ou six heures, Ils se croient les Premiers Hommes du monde.?

Il y a là un véritable réquisitoire contre la vie mondaine, faite d'amusements littéraires et de commérages insipides, au lieu de

9 ISaint-Hyacinthel, Entretiens dans lesquels on traite des entreprises de l'Espagne..., La Haye, A. de Rogissart, 1719, p. 89-91. On peut voir aussi clans son. travail de journaliste le sérieux de ses recherches et de ses commentaires plutôt que le goût du petit commérage. D'où, par exemple, telle critique, en février 1718 dans l'Europe savante, du Nonveau Recueil de Pieces Fugitives d'Histoire, de Litterature, etc., par l'Abbé Archimbaud, Paris, 1717: "il nous parôt aussi qu'il cherche plûtôt à recueillir des Nouvelles, qu'à s'instruire exactement des choses dont il parle." (p. 269). 
fournir réflexions et savoirs. La suite du texte cherche bien à asseoir toute analyse sérieuse sur une juste et savante recherche des principes de gouvernement. Même si, au bout du compte, l'idée est de justifier la politique du Régent, la tentative de la fonder en raison et d'en saisir la justice conduit, paradoxalement, à suivre de près Grotius et à s'appuyer sur des principes du genre: "La Nature faisant naitre tous les Hommes égaux, nul Homme n'a donc naturellement un Droit de propriété sur un autre Homme, de sorte qu'il puisse en disposer à sa volonté"; ou encore: "si la nécessité a forcé les Hommes à se réunir sous de certaines Loix, pour former des Sociétez qui assurassent leur bonheur; ces mêmes Hommes ont toujours le Droit de former entre eux de nouvelles Loix, lorsqu'ils le jugent nécessaire; parce que ne s'étant réunis que pour être heureux, leurs vûes subsistant toujours, ils ont toujours le même Droit de travailler à y parvenir. " ${ }^{10}$ C'est pourquoi il est possible de rééditer le texte en 1733 , alors même que le contexte historique a changé, car la valeur des arguments et des principes demeure évidente, d'autant que, sur certains points, ils anticipent sur Montesquieu qui leur donnera encore plus d'autorité.

La critique de la mondanité n'empêche cependant pas SaintHyacinthe de participer activement à la vie des salons. SaintHyacinthe n'est pas Rousseau. La satire porte surtout sur les petitsmaitres et les jolis marquis, non sur la vie mondaine comme telle. Le bonheur d'une existence tient à la juste alternance entre l'enjouement et la légèreté mondains et le sérieux et l'application d'une réflexion savante: l'idéal encore d'un Diderot. SaintHyacinthe est, en particulier, un familier du salon de Mme de Lambert et il en demeurera, semble-t-il, fort proche. Il trouve, en même temps, un charme à la vie contemplative et au calme de la retraite. En Hollande, il publie en 1722 des Lettres écrites de la campagne où il tente de faire valoir une philosophie morale fondée sur des principes laïcs. Là encore, succès dans la République des Lettres (la Bibliothèque ancienne et moderne, après un compte rendu élogieux, en fournit des extraits longs de sept pages, contre deux pages accordées à d'autres lettres qui viennent d'être publiées la même année: les Lettres persanes de Montesquieu!).

Quant à son mariage qui le place dans une position sociale et financière plus assurée, loin d'être l'aventure de la légende, il

10 Ibid., respectivement p. 232 et p. 211. 
apparaît que ce fut un mariage validé par la présence des parents et la bienveillance des autorités (même si la famille de sa femme est profondément protestante et lui, au mieux, déiste). Parvenu en Angleterre, dans un climat politique qui lui convient mieux que la France des années 1720 , il est reçu aussi bien dans la bonne société londonienne qu'à la Royal Society (où il parrainera d'ailleurs en 1730, derechef, Montesquieu!). Ses pertes financières et la désobligeance de Georges II pour les émigrés protestants l'obligent, certes, à quitter précipitamment Londres en 1732, mais c'est pour mieux retrouver la vie parisienne et les salons de Mme de Tencin et de la duchesse du Maine. Il en profite pour, sous couvert de contes de fée, faire une satire de Georges II et une apologie du prince de Galles (Le prince Titi, 1735) où la badinerie critique s'allie au bréviaire du juste gouvernement pour produire une pièce à succès qu'une seconde édition, l'année suivante, prolonge.

Si ses dernières années, en raison de son exil forcé, de ses finances de plus en plus serrées et de la mort de son épouse (avec qui il a formé un couple, à ce qu'il semble, fort heureux: ce qui n'est pas l'habitude dans les mariages au XVIII ${ }^{\circ}$ siècle) ne concordent plus avec une carrière mondaine et savante parfaitement menée, il n'en demeure pas moins que Saint-Hyacinthe a connu gloire et aisance, que ses contemporains l'ont reconnu comme un personnage notable de la République des Lettres et qu'il semble tout à fait abusif de l'inscrire dans la marginalité et la bohème littéraire.

Nous voici donc en présence de deux histoires dont il serait facile de prétendre que la seconde, historiquement plus sérieuse, attentive à débusquer légendes et commérages, potins et racontars, est la plus fiable. Je voudrais ici ne pas céder à cette facilité. Dans ce genre d'opérations, il s'agit bien souvent d'acquérir le privilège institutionnel de découvrir un génie méconnu. Or SaintHyacinthe n'est pas un Montesquieu oublié. S'il a de nombreuses qualités, s'il occupe une position importante parmi les seconds couteaux de la vie littéraire, il serait par trop réducteur de ne plus voir en lui que la réussite sociale et la vivacité intellectuelle. Je crois qu'il est bon de prendre les deux histoires au sérieux et de saisir, du coup, ce qu'elles révèlent dans leur opposition même.

Un premier élément que l'on peut en tirer est l'idée que le maillage du filet institutionnel est, à l'époque, suffisamment large pour permettre de circuler, sans césure forte, de l'ombre des 
marges à la lumière des pleines pages. Ou, plus exactement, cela permet de révoquer l'idée qu'existe, au début du XviI' ${ }^{\mathrm{e}}$ siècle, une "marginalité" littéraire au strict sens du terme. Non seulement l'institution tolère sans peine une dissémination qu'elle ne saurait, de toute façon, contrôler, mais surtout il ne vient à l'esprit de personne de tenter de jouer d'une position marginale pour mieux valider la justesse (et la justice) de sa posture: Rousseau n'est pas encore venu et nous sommes encore plus loin des mouvements d'avant-garde qui feront leur beurre symbolique en refusant de se changer en crème de la crème de l'establishment.

Le second point tient à ce travail de la médisance qu'on ne saurait ramener à un simple exercice de délire social ou de jalousie d'auteur, car ce serait ignorer la valeur communautaire du commérage. Le potin définit un espace social qui, dans la vie mondaine et, même, dans la République des Lettres joue un rôle important. Il n'a certes pas cessé d'ordonner une partie de nos existences, mais il occupe une position beaucoup plus cruciale dans le monde de l'Ancien Régime. On pourrait dire, d'une formule, qu'au $\mathrm{XvII}^{\circ}$ siècle, on passe d'une pragmatique du potin à une esthétique de la marge. Autrement dit, d'une présence de l'oral qui noue, selon le modèle de la conversation, des échanges entre interlocuteurs, on transite vers une représentation de l'écrit (qu'indique le terme même de "marge") qui impose une dissymétrie radicale entre producteurs et récepteurs. Là où il fallait souscrire à un principe pragmatique d'échange (la seule façon de recevoir un potin est d'en donner un autre) selon une dimension temporelle, on doit désormais produire une expérience esthétique (donc individuelle) selon une dimension spatiale où les jeux de positionnalité dans un champ donné prennent le dessus. À la joute oratoire (implicite) s'oppose le principe même de l'opposition, c'est-à-dire la recherche de la différence pure. La possibilité d'échange et de circulation est justement ce qui assure la polygraphie et permet l'hétéroclite des occupations et des discours, alors que la volonté de différenciation impose une homogénéité du lieu occupé, voire, bientôt, une paranoïa de la position. D'un côté, parcellisation et affrontement; de l'autre, échange et tact. Le tact est, en effet, central dans la vie mondaine: c'est la politesse du toucher, espace du rhétorique qui parvient à allier docere (enseigner) et delectare (plaire, amuser), justement parce qu'il développe un sens du movere (émouvoir, toucher). Cette théâtralité du rhétorique propre à la politesse mondaine (et, dans une mesure 

. 
34

valeur et en saisit la dimension propice à la nouvelle situation de l'écrivain:

Je félicite le Public de l'envie qui m'a pris d'être Auteur, il aura le Livre le plus varié du monde, \& de plus un Livre qui ne finira jamais. Ainsi on goûtera le plaisir charmant de la variété, sans craindre de le voir finir. Un plaisir qui dure toujours, n'est-il pas le plus grand de tous les plaisirs?

Le Titre seul aura fait sentir au Lecteur intelligent la vérité de ce que je viens de dire. Il l'annonce parfaitement. C'est le plus beau Titre qu'on puisse choisir. Il dit par un seul mot quel est le but, le dessein \& le sujet \& la manière de le traiter; \& fait en même tems connoitre, que le sujet est immense, le dessein inépuisable, la manière aisée \& que le but dans son unité renferme une variété infinie: semblable à l'Univers qui n'est qu'un clans l'assemblage d'un nombre innombrable d'êtres, toujours le même \& toujours varié, toujours ancien \& toujours nouveau: quel terme en effet peut-on assigner à la fantaisie de l'Homme? Tout ce qui est, \& tout ce qui n'est pas, peut en être l'objet, souvent même ce qui ne peut pas être. Ainsi quand la fantaisie me prendroit de ne plus écrire, ou que ma mort qui n'est pas éloignée, me feroit discontinuer cet Ouvrage, parce que j'aurai alors d'autres choses à faire [!], cet Ouvrage ne discontinueroit pas pour cela: il se trouvera toujours assés d'Ecrivains fantasques tout prêts à le continuer, après eux d'autres encore, auxquels d'autres encore succederont $\&$ ainsi in secula seculorum, amen. C'est sur quoi j'ai fondé sa durée infinie, ainsi que j'en ai fondé le plaisir sur la variété. (p. 1-2)

La nouvelle et bien sarcastique religion qu'il invente est celle des Lettres et de la pure fantaisie, alliage de la légèreté mondaine et de l'ouverture sérieuse à un univers qui semble toujours plus vaste. La tradition et la transmission d'un héritage y trouvent encore demeure, mais assignées à la variété infinie plus qu'à la répétition. Puisque l'homme apparaît si nécessairement fantasque, comment forclore la figure sociale de l'auteur à des tâches et à des légitimations ordonnées par la tradition, les patrons ou les institutions littéraires? Nous avons là une vision de ce que Daniel Roche nomme "phénomène de consommation culturelle" et dont il examine les débuts au XVIII ${ }^{c}$ siècle ${ }^{17}$. Le désir d'être auteur suffit à son auto-légitimation, mais pareil désir s'instaure d'avoir un

17 Daniel Rochc, Les Républicains des lettres: Gens de culture et Lumières au xvir" siecle, Paris, Fayard, 1988, p. 21. 
public qui le réclame. Quoi d'autre pourrait justifier de se vanter du nom d'auteur?

En lisant la première Epitre du second livre des Epitres d'HORACE, je trouvai qu'il y remarquoit, "qu'un homme qui n'avoit pas apris la navigation, n'entreprenoit pas de conduire un Vaisseaul qu'on n'osoit donner à un malade un remède, qu'on ne savoit préparer; qu'en un mot personne n'exerçoit un métier sans l'avoir apris, excepté celui de faire des vers, dont les ignorans \& les savans se mêlent indifféremment. [...] Je fis réflexion qu'au lieu de borner cette remarque aux faiseurs de vers, on pouvoit en général l'étendre aux faiseurs de Livres. Se mêle d'en faire qui veut s'en mêler. On ne fait d'apprentissage que pour les imprimer, les relier ou les vendre, on n'en fait point pour les composer. En effet rien n'est plus facile, on n'a pas besoin d'aprentissage. On dit que pour jouer de la flute, il ne faut que soufler \& remuer les doigts; on peut dire de même que pour faire un Livre, il ne faut que penser \& écrire. Cela est vrai, dira quelqu'un, mais il faut savoir bien penser \& bien écrire. [...] Songez-vous, MONSIEUR le dificile, que parmi plus de trois ou quatre cent mille volumes imprimés depuis l'invention de l'Imprimerie, il n'y en a peut-être pas dix qui soient tels que vous voulez que soit un bon Livre; pas dix, il n'y en a peut-être pas quatre, si on en excepte quelques petits traités particuliers, si petits qu'ils ne sont presque que des feuilles volantes? Si on ne donnoit au Public que des Livres tels que vous les souhaitez pour être bons, que deviendroient tant de femmes lors qu'elles se trouveroient sans compagnie dans l'intervale de leur toilette à leur dîner, ou de leur dîner à leur Quadrille? Voudriez-vous qu'elles ne fissent que baailler [sic] seules, ou en présence d'un mari qui les ennule, ou que dans l'impatience de voir quelqu'un qui les rejouiroit, elles fussent tourmentées de vapeurs? Les Livres nouveaux préviennent ces inconveniens, ils les amusent, \& après les avoir amusées, ces Livres vont dans l'Antichambre servir d'amusement à ceux des Laquais qui aiment la lecture $\&$ les empêchent de jouer aux cartes. Mais que deviendroient même dans leurs momens d'ennui tant de Courtisans, de Guerriers, de Financiers, de Gens de Robe, de Marchands quand les chalans leur manquent; tant d'Abbés qui ne savent à quoi s'occuper, tant de beaux esprits qui ne savent que critiquer, en un mot tant d'honnêtes gens qui s'ennuïent [...] J'en reviens à ceci, c'est qu'à juger par les effets, nous n'avons point de Livres tels que vous voulez qu'ils soient. En attendant modérons un peu votre définition de bien penser $\&$ de bien écrire; s'il faloit s'y conformer, je n'oserois me faire Auteur, \& je meurs d'envie de l'être. Ce ne seroit peut-être pas 


38

Saint-Hyacinthe ne cherche jamais la marginalité, mais il trouve, pendue à sa porte, l'originalité, par où la tradition et l'autorité du passé ne peuvent plus jouer pour induire comportements, postures et œuvres. La création du fantasque est déjà plaisir de l'autonomie. Mais rien de revendicatif en cela, rien non plus de vindicatif. Loin des ruptures et des avant-gardes, loin des volontés ou des désirs de la marginalité, il s'agit d'articuler, avec le plus de jouissance possible, la vivacité du bel esprit et la liberté du philosophe (là où Voltaire, à l'inverse, s'acharne à les vouloir différencier) ${ }^{19}$ :

À l'égard du caractère original qu'on a trouvé dans mes Fantasques, on devait s'y attendre, le titre seul l'annonce. Qu'y a-t'il de plus original que la fantaisie. C'est une source féconde de choses auxquelles ne s'attend pas celui-même qui les écrit; c'est un mouvement bizarre qui le porte où il ne sait pas qu'il va, \& où il ne croit point aller. Toujours libre, sans règle, sans objet que celui de se livrer à soi-même, il faut bien que les écrits d'un Fantasque ayent un caractère original. Comment ne l'auroient-ils pas? La fantaisie qui les dicte est quelque chose de si original, qu'il semble qu'elle ne doive son origine qu'à ellemême. [...] Pour ces fantaisies qi se laissent conduire par la coûtume \& par l'exemple, ce sont de ces pauvres fantaisies qui n'ont jamais un caractère original; elles sont communes, elles n'ont rien de singulier. Elles se prêtent aux préjugés, elles se conforment aux opinions reçuës: la vérité leur fait peur si elle n'est munie de certificats \& d'un passeport. Au lieu qu'une fantaisie libre, courageuse, qui se détermine par elle-même, s'attache à ce qui lui plait $\&$ ne s'y attache que parce que cela lui plait. C'est ce qui donne ce caractère original qu'on a trouvé dans mes Fantasques \& que j'y conserverai s'il plait à ma fantaisie. (p. 180)

Saint-Hyacinthe, parce qu'il allie, dans son existence comme dans ses productions, badinage de salon, érudition historique et critique sociale, apparaît atypique, parce que toujours en porte-àfaux et par rapport aux traditions érudite et mondaine et par rapport à la philosophie qui se met en place à l'époque, mais il semble aussi typique de ces déplacements qui touchent au $\mathrm{XVIII}^{\mathrm{e}}$ siècle auteur et public, commerce et savoir, potinage et réflexion. L'idéal semble, chez lui, devenir celui d'un bavardage philosophique qui empêche toute fixation dans un état ou dans

19 Voir l'article "Bel esprit " dans l'Encyclopédie rédigé par Voltaire. 
une opposition. Il est sans doute symptomatique que, dans son conte de fée politique, l'Histoire du Prince Titi, les amoureux que sont le jeune prince et la princesse Bibi ont reçu le privilège, grâce à la Fée Diamantine, de se changer en quelque animal qu'ils voudront, et qu'au moment où il devient roi, Titi ne puisse plus se changer en oiseau pour aller voir sa bien-aimée, car "il ignoroit que le don de se métamorphoser cessoit lorsqu'on étoit passé d'un état de vie incertain, à un état fixe. " ${ }^{20}$ C'est à cette incertitude qu'il faut se tenir - au moins pour y gagner un peu d'atypicité dans le monde savant et bien rempli de certitudes qui est le nôtre.

20 Histoire du Prince Titi, Paris, Veuve Pissot, 1736, p. 87. 